\title{
Enseñanza del periodismo transmedia en Colombia, una experiencia pedagógica con estudiantes universitarios
}

\author{
Teaching Transmedia journalism in Colombia, a pedagogical \\ experience with university students
}

\section{Ensino do jornalismo transmídia na Colômbia, uma experiência pedagógica com estudantes universitários}

\begin{abstract}
Nelson Nieto Borda, Universidad de Bogotá Jorge Tadeo Lozano, Bogotá, Colombia (nelson.nietob@utadeo.edu.co)
\end{abstract}

RESUMEN | Este artículo socializa la sistematización de la experiencia pedagógica de la enseñanza del periodismo transmedia con base en el proceso de Aprendizaje Basado en Problemas (ABP), utilizado con estudiantes de periodismo de la Universidad de Bogotá Jorge Tadeo Lozano en los años 2016 y 2017. En su abordaje, los estudiantes trabajaron en la propuesta, investigación, producción y publicación de contenidos periodísticos, un ejercicio que tuvo como objetivo principal facilitar la comprensión teórica y práctica de los procesos convergentes, la producción de narrativas periodísticas y la interacción con las audiencias. La sistematización se sustenta en la observación de once proyectos en las etapas de conceptualización, producción de medios y contenidos a nivel narrativo y tecnológico, así como la gestión de redes sociales y la realización de actividades en escenarios no digitales. Asimismo, se basa en la valoración de la percepción de aprendizaje otorgada por los estudiantes de los grupos de proyecto. Estas observaciones permiten afirmar que el ABP resulta clave para lograr un aprendizaje significativo e integral; este método desarrolla habilidades en materia de estrategia, producción técnica y periodística para la multiplataforma, y otorga a los estudiantes competencias para relacionarse con sus audiencias. Del mismo modo, evidencia que el periodismo transmedia, desde la academia, necesita ser complementado y articulado sinérgicamente con otras propuestas y espacios mediáticos de formación, más allá del salón de clase. Este documento aporta elementos a considerar en la configuración de estrategias educativas en la enseñanza-aprendizaje del periodismo transmedia.

PALABRAS CLAVE: periodismo transmedia; periodismo convergente; periodismo digital; aprendizaje basado en problemas; generación de contenidos; storytelling.

\section{FORMA DE CITAR}

Nieto-Borda, N. (2021). Enseñanza del periodismo transmedia en Colombia, una experiencia pedagógica con estudiantes universitarios. Cuadernos.info, (48), 215-236. https://doi.org/10.7764/cdi.48.27827 
ABSTRACT / This article socializes the systematization of the pedagogical experience of teaching transmedia journalism based on the Problem-Based Learning (PBL) process, used with journalism students from the Universidad de Bogotá Jorge Tadeo Lozano in 2016 and 2017. In its approach, the students worked on the proposal, research, production and publication of journalistic content, an exercise whose main objective was to facilitate the theoretical and practical understanding of convergent processes, the production ofjournalistic narratives and interaction with audiences. The systematization is based on the observation of eleven projects in the stages of conceptualization, production of media and content at a narrative and technological level, as well as the management of socialnetworks and the performance of activities in non-digital settings. Likewise, it is based on the assessment of the learning perception given by thestudents of the project groups. These observations allow us to affirm that PBL is key to achieving meaningful and comprehensivelearning; this method develops skills in terms of strategy, technical and journalistic production for the multiplatform, and gives students assets to relate to their audiences. In the same way, it shows that transmedia journalism, from the academy, needs to be complemented and articulated synergistically with other proposals and media training spaces, beyond the classroom. This document provides elements to consider in the configuration of educational strategies in the teaching-learning of transmedia journalism.

KEYWORDS: transmedia journalism; convergent journalism; digital journalism, project-based learning; content generation; storytelling.

RESUMo | Este artigo socializa a sistematização da experiência pedagógica do ensino do jornalismo transmídia com base no processo de Aprendizagem Baseado em Problemas $(\mathrm{ABP})$, utilizado com estudantes de jornalismo da Universidade de Bogotá Jorge Tadeo Lozano nos anos de 2016 e 2017. Na nossa abordagem, os alunos trabalharam na proposição, pesquisa, produção e publicação de conteúdos jornalísticos, um exercício que teve como objetivo principal facilitar a compreensão teórica e prática dos processos convergentes, a produção de narrativas jornalísticas e a interação com públicos. A sistematização baseia-se na observação de onze projetos nas fases de conceituação, produção de meios e conteúdos a nível narrativo e tecnológico, bem como a gestão de redes sociais e a realização de atividades em contextos não digitais. E, em segundo lugar, na avaliação da percepção da aprendizagem conferida pelos alunos das turmas do projeto. Essas observações que permitem afirmar que a $A B P$ é fundamental para alcançar uma aprendizagem significativa e integral que desenvolva habilidades em termos de estratégia, produção técnica ejornalística para a múltiplataforma e na forma como os alunos buscam se relacionar com seus públicos. Da mesma forma, mostra que o jornalismo transmídia, desde a academia, precisa ser complementado e articulado sinergicamente com outras propostas e espaços de formação midiática fora da sala de aula. Este documento fornece elementos a serem considerados na configuração de estratégias educacionais no ensino-aprendizagem do jornalismo transmídia.

PALAVRAS-CHAVE: jornalismo transmídia; jornalismo convergente; jornalismo digital; aprendizagem baseado em problemas; geração de conteúdos; storytelling. 


\section{INTRODUCCIÓN}

En Colombia, a partir de la segunda década del siglo XXI, los conceptos de convergencia mediática y narraciones transmediáticas empezaron a cobrar fuerza en la medida en que el mundo y el país experimentaban cambios tecnológicos importantes y, en consecuencia, un crecimiento significativo de usuarios conectados a Internet. De acuerdo con estudios oficiales, para 2010, la población colombiana ascendía a 45,5 millones de habitantes (DANE, 2017), de los cuales el $37 \%$ ya eran usuarios de Internet (Banco Mundial, 2017). Para enero de 2020, la población aumentó a 50,6 millones, siendo un 69\% usuaria de la red. Esto quiere decir que, en el lapso de nueve años, la cantidad de colombianos conectados pasó de 16,8 millones a 35 millones, crecimiento impulsado de manera notoria por la tecnología móvil, que en términos de conexiones versus el total de población asciende a $119 \%$, un millón de conexiones más respecto de enero de 2019 (Datareportal, 2020). Según el Ministerio de las Tecnologías de la Información y las Comunicaciones, para el cuarto trimestre de 2019 el país superó los 66,2 millones de abonados (Mintic, 2020).

Según Datareportal, los usuarios de 16 a 64 años se conectan a Internet más de nueve horas al día, de las cuales dedican casi cuatro en el uso de medios sociales, tres horas y media a ver televisión y otro periodo semejante a escuchar música vía streaming. El estudio muestra que en su respectivo orden YouTube, Facebook, WhatsApp, Instagram, Facebook Messenger, Twitter, Pinterest, LinkedIn, Skype y Snapchat son los diez medios más accedidos por los usuarios colombianos (Datareportal, 2020). En la Primera Gran Encuesta TIC / 2017 (Mintic, 2017) que el gobierno de Colombia realizó -y en la que se asegura que $72 \%$ de los hogares colombianos lograba acceder a Internet a través de un smartphone - se lograron determinar las prácticas que los hogares conectados le daban a dichas tecnologías. En esta encuesta, 94\% de los hogares manifestaron que emplearon Internet para comunicarse, 78\% para entretenerse y $66 \%$ para informarse. Asimismo, $79 \%$ lo usa para acceder a redes sociales, 70\% para uso de mensajería, 67\% para correo electrónico (Mintic, 2017), siendo Facebook el medio social más usado (88\%), seguido por WhatsApp (87\%), y sucesivamente por Instagram (34\%), Google $+(29 \%)$ y Twitter (20\%).

Con este ecosistema mediático cada vez más diverso y transitado se hicieron todavía más evidentes los procesos de convergencia en la multiplataforma y la proliferación de novedosas formas de producción narrativa transmediática, lo que ha motivado a que desde la academia se profundice en la comprensión del fenómeno mediante bibliografía reciente, el análisis casuístico, el debate y la experimentación con esta narrativa en el ámbito de la no ficción sobre temas de coyuntura, a fin de que esta pueda ser compartida en las aulas con los estudiantes. Por esta razón, la pregunta que movilizó este ejercicio apuntó a cómo configurar y moldear el proceso de aprendizaje de los estudiantes universitarios en periodismo, transmedia, y al 
desarrollo de habilidades instrumentales de producción, mediante experiencias periodísticas reales, entendido esto también como el aprendizaje al servicio de la resolución de problemas sociales, cuya producción de sentido es sometida a las reglas del actual ecosistema mediático y narrativo.

En este sentido, desde un enfoque de la comunicación digital, se buscó comprender los procesos convergentes y los aspectos que caracterizan a la narrativa transmedia como una tarea que implica hacer una reflexión y un análisis sobre el rol que juegan las tecnologías de la información y de las comunicaciones (TIC) como soporte instrumental que dinamiza y acentúa dichos procesos. Aunque la narrativa transmedia tiene como punto de partida historias que se generan desde cualquier plataforma mediática, por correspondencia con la naturaleza de la asignatura de desde la cual se trabajó la propuesta, se planteó en el contenido programático la construcción de una narrativa hipermedia (Moreno, 2012) articulada por un sitio web que funcionara como punto de concentración de contenidos y como una forma, entre varias, de abordar el proyecto periodístico transmedia.

Con el propósito de contribuir al proceso de formación en las condiciones anteriormente señaladas, se optó por abordar una didáctica de Aprendizaje Basado en Problemas (ABP), en donde se articula la conceptualización teórica, la apropiación instrumental, la construcción narrativa y la gestión de contenidos. En consecuencia, este ejercicio académico evidencia la experimentación de una metodología pedagógica integral con el objeto de fortalecer las competencias profesionales de los estudiantes pertinentes al contexto mediático y laboral del mundo actual. Por ello, la investigación aplica la sistematización de experiencias educativas para recuperar la experiencia vivida, representada en nuevos conocimientos que permitan desarrollar y estructurar de manera más eficiente la propuesta académica; asimismo, busca aportar más elementos a la comunidad docente que enseña en esta área de conocimiento, y ha sido relevante para desarrollar el componente convergente en el nuevo plan de estudios del programa.

Según lo anterior, este trabajo da cuenta, en un primer momento, de las bases teóricas que permiten comprender el escenario conceptual en la relación convergencia, transmedia y periodismo. A nivel metodológico, desglosa y explica las etapas que se emplearon como modelo para configurar el derrotero de una producción transmedia desde el aula. En la misma línea, detalla los ejes sobre los cuales se concentra la sistematización de la experiencia. En cuarto lugar, presenta los resultados del trabajo desarrollado por los estudiantes, poniendo en común los principales aspectos hallados en ellos, los que permiten observar el cumplimiento de los objetivos de aprendizaje y la valoración y percepción de los alumnos frente al proceso. 


\section{MARCO TEÓRICO}

\section{Periodismo transmedia y el Aprendizaje Basado en Problemas (ABP)}

Los procesos de convergencia mediática (Jenkins, 2008) que hoy se presentan en el actual ecosistema mediático (Scolari, Manuel, \& Mar, 2012), dan lugar a diferentes formas narrativas, como las construcciones crossmedia (Costa \& Piñeiro, 2012) y la transmedia (Scolari, 2013), narrativas que también se ven influidas por la creciente cultura participativa que se desarrolla en el mencionado ecosistema (Aparici, García-Marín, \& Díaz-Delgado, 2019).

De acuerdo con las fuentes, las narrativas transmedia (Scolari, 2016) se pueden entender como aquellas historias que se cuentan a través de diferentes medios y plataformas, empleando distintas configuraciones mediáticas de naturaleza digital, análoga, o ambas, en donde la audiencia juega un rol importante como fuerza creadora (López-Carril, Villamón-Herrera, \& Añó-Sanz, 2019) y, por lo tanto, coautora del universo narrativo en la medida en que son los receptores, en su condición activa, los que tienen la capacidad de generar nuevas expansiones al relato principal.

Dicha condición activa dinamiza la relación entre todos los actores, dando lugar a procesos de colaboración, ya sea para el mejoramiento de la red, para participar en su construcción, o específicamente para conectar con el mundo técnico, profesional y conceptual del periodismo y los medios de comunicación en su conjunto, logrando paralelamente la visibilidad y empoderamiento de dichas personas y de las organizaciones que representan (Camargo, Vieira Miranda, \& Magnon, 2019). Por lo tanto, una forma de comprender el periodismo transmedia es entendiéndolo como una forma de lenguaje compuesto de otros lenguajes y narrativas (Renó \& Flores, 2018) generadas por los periodistas y las personas o grupos que se interesan por aportar sus informaciones y discernimientos a las demás audiencias. Se puede entender como una construcción de historias sin agenda única de producción, con un propósito común que consiste en aportar y compartir lo que es de interés público por su carácter novedoso, humano o insólito.

Estas son las nuevas generaciones de consumidores de información (Scolari \& Establés, 2017) de la cultura participativa que no se quedan con lo que les entregan, sino que se dan a la tarea de explorar, cazar y recolectar datos de una manera más comprometida, llegando a conectar elementos que no fueron abordados total o parcialmente por el autor inicial. Se trata de una nueva relación que ha podido evidenciar que el ejercicio periodístico está sufriendo cambios importantes y que se generan nuevas formas de interacción entre el periodista, los medios y la audiencia (Rost \& Bergero, 2012), en donde no existe la simple exposición de 
noticias, sino el establecimiento de nuevas reglas que recuperan y privilegian las distintas formas de interacción.

Por lo anterior, la enseñanza del periodismo transmedia exige explorar y experimentar con nuevas metodologías y formas de creación, que prospecten -y que se practiquen sobre- las realidades de tal diversidad mediática, sus composiciones narrativas y las características del sujeto que habita y convive en el ecosistema mediático actual. Lo anterior, con el propósito de ejercitar en los estudiantes de periodismo su capacidad estratégica y ejecutiva como creadores y gestores de experiencias periodísticas. El sujeto de la transmedia es el prosumidor (Sarsa, 2014) y presenta una condición activa de consumidor, colaborador y productor de contenidos a través de las tecnologías digitales basadas mayoritariamente en la Web 2.0, habilitadas para la creación, edición, publicación y gestión de contenido entre todos los miembros del circuito mediático.

Dadas estas reglas de juego, el periodista, en tanto productor transmedia, ha de comprender que su trabajo creativo no recae solo en el medio y estilo narrativo que imprime a su contenido, sino que plantea el diseño de experiencias integrales con las que el mensaje ha de ser revelado a la audiencia (Pratten, 2015). Así, ha de tenerse en cuenta el uso pertinente y estratégico de cada contenido según su plataforma, la interactividad prevista para sus públicos objetivo, sus etapas de maduración y la medición de resultados en cada una de ellas (Vázquez-Herrero, González-Neira, \& Quintas-Froufe, 2019).

Así, es importante que las experiencias periodísticas puedan proponer la forma en la que sus consumidores pueden expandir la narrativa, causar la inmersión e inspirar a la acción, entre otras características (Scolari, 2013; Nieto, 2017). En su conjunto, y sin pretender condicionar en forma alguna la composición narrativa transmedia, el principal interés radica en lograr convocar la participación de la audiencia y desafiar su capacidad creativa.

Por ello, esta propuesta pedagógica se propone enfrentar los desafíos de la actual realidad mediática en el campo del periodismo. Así, el diseño de la asignatura estructuró un espacio académico de reflexión y práctica, para permitirle al estudiante la comprensión del momento histórico de los medios y, a su vez, desarrollar las destrezas profesionales que exige el mundo laboral en la materia, a partir de un currículo armónico con las teorías y la práctica para dar solución a las problemáticas comunicacionales y sociales de coyuntura.

Esta apuesta busca fortalecer lo que se conoce como la competencia mediática (Pérez-Rodríguez, 2020), un concepto que implica superar la idea básica de instrumentalización o la simple decodificación fomentando otras capacidades 
que garanticen procesos de lectura y escritura mediática, en contextos semióticos y pragmáticos más amplios, especialmente el cultural y el de la interacción social, implicando distintas habilidades y formas de alfabetización (p. 25).

En consecuencia, se aplica el modelo de Aprendizaje Basado en Problemas (Méndez Urresta, Méndez Urresta, \& Méndez Carvajal, 2017) como estrategia didáctica viable para que el estudiante logre obtener los conocimientos y la experticia que propone la asignatura. El ABP, como también se le conoce, permite enfrentar de manera innovadora e interactiva la resolución de problemas complejos de la realidad (Casa, Huatta, \& Mancha, 2019) desde un ambiente académico mediante un proyecto de trabajo en el cual los grupos de trabajo definen un tema, plantean preguntas, las debaten, recolectan datos, los analizan, reflexionan sobre su aprendizaje, y generan conclusiones y productos para luego socializarlos.

Para la enseñanza del periodismo transmedia, el ABP resulta viable en la medida en que el estudiante consigue una mayor motivación emocional, retiene de mejor manera el conocimiento, estimula su pensamiento crítico creativo, aumenta sus habilidades interpersonales, promueve la evaluación formativa y, sobre todo, en el marco de su proyecto de clase, logra mayor integración del conocimiento al identificar en su propuesta las diferentes teorías, disciplinas, componentes, procesos y procedimientos propios de su desarrollo (Luy-Montejo, 2019).

En este ejercicio, el estudiante puede obtener un aprendizaje significativo que se entiende como la integración exitosa de nuevos conocimientos en su estructura cognitiva (Carranza \& Caldera, 2018) en medio de una dinámica que lo invita a trabajar en equipo de manera colaborativa, a asumir responsabilidades, a enfrentar desafíos, a explotar su potencial creativo, a desarrollar la capacidad crítica y comunicativa, a experimentar con los objetos de estudio, a conectar la academia con la realidad, animándolo a decidir sobre la forma con la que obtendrá respuestas para la solución de los problemas planteados (Maldonado-Pérez, 2008).

De acuerdo con lo anterior, el ABP supone replantear el aprendizaje tradicional dependiente de la memoria y la repetición toda vez que conecta al estudiante con la adopción de un enfoque interdisciplinar y cooperativo para el abordaje de las complejidades sociales, que en la práctica le invitan a aumentar su implicación con el entorno y, en consecuencia, a perseverar el logro de sus intereses. Por último, es necesario señalar que el ABP ya ha sido probado y promovido para la formación de periodistas, particularmente del ciberperiodismo, en donde los resultados han sido satisfactorios y se plantean nuevas propuestas para trabajar cooperativamente desde distintos contextos culturales y educativos (Larrondo et al., 2020). 


\section{METOdOLOGÍA}

Esta investigación es cualitativa y aplica la sistematización de experiencias educativas (Bermúdez, 2018) como metodología que propende por la producción de conocimientos de manera permanente y acumulativa, tomando como base la recuperación, reconstrucción y análisis de experiencias vividas en las intervenciones realizadas sobre una realidad social para rescatar y compartir los aspectos más relevantes y significativos, en este caso del proceso de formación de estudiantes en periodismo transmedia.

La estrategia pedagógica se articuló a través de la didáctica del ABP y planteó a los estudiantes pertenecientes a las cohortes del segundo semestre de 2016 hasta el segundo 2017 de la asignatura de Comunicación y Nuevas Tecnologías II, del Programa de Comunicación Social - Periodismo de la Universidad de Bogotá Jorge Tadeo Lozano, la proposición y definición de un proyecto periodístico transmedia delineando un trayecto en donde los jóvenes pudieran transitar por tres etapas: conceptualización, creación de medios y contenidos periodísticos, y gestión de contenidos en medios sociales. Sobre esta base se edificó una propuesta desde el campo de la comunicación digital como una aproximación a la generación de contenidos de periodismo transmedia en el marco de los procesos creativos y multifacéticos de la convergencia (Villanueva-Mansilla, 2017).

\section{Estructura de la asignatura en el marco del ABP}

La propuesta pedagógica que integra el ABP en la asignatura se compuso de tres etapas que, en un lapso de 16 semanas, con un desarrollo paralelo y de distinto grado, combinó la teoría y la práctica. El proyecto que se articuló en el marco de esa propuesta pedagógica fue concebido para que el estudiante tuviera la capacidad de desarrollar el pensamiento estratégico para así poder estructurar una narrativa transmedia (multiplataforma), que instara a las audiencias a lograr mayor involucramiento, a practicar el reporteo y la producción conceptual de contenidos periodísticos, y a explorar y apropiar aplicaciones de la Web 2.0 destinadas a la creación, edición, publicación y gestión de contenidos en sitios web y en medios sociales.

\section{Etapa 1. Conceptualización}

Desde la parte teórica de la convergencia se realizó una aproximación a la definición, caracterización y reconocimiento del actual ecosistema mediático, así como a los procesos de convergencia mediática en sus distintas dimensiones, y se identificaron las distintas formas narrativas presentes en este escenario y, en particular, las que corresponden al periodismo. Se realizó un abordaje teórico a partir de material bibliográfico básico que permitiera a los estudiantes complementar los conceptos entregados en clase. 
Etapa 1

Semana 1 a 5

Proposición de tema.

Aprobación en consejo de redacción.

Investigación periodística

Registro multimedios.
Etapa 2

Semana 6 a 10
Etapa 3

Semana 11 a 16

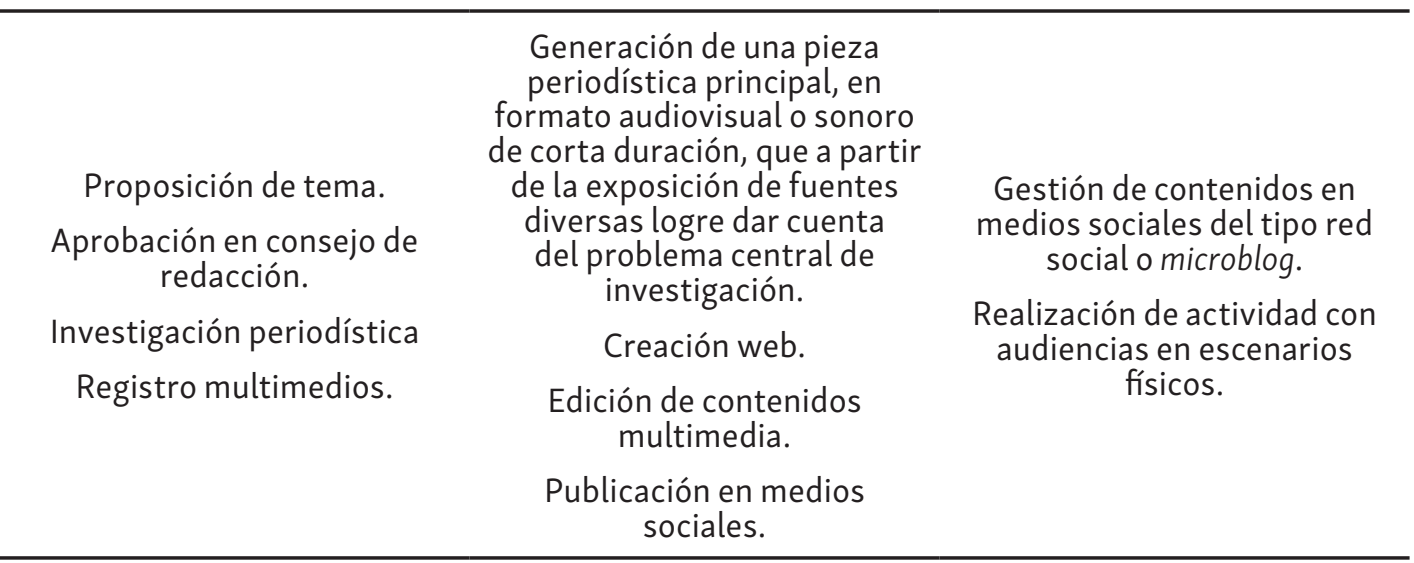

Tabla 1. Actividades programadas para cada etapa del proyecto

Fuente: Elaboración propia.

En el componente práctico de esta etapa, los estudiantes dedicaron la mayor parte de su trabajo autónomo en casa para preparar la propuesta temática de un proyecto periodístico. El tema debía ser acotado y con posibilidad de acceder sin dificultad a fuentes y locaciones. Para la presentación de sus propuestas los estudiantes desarrollaron un formato que contenía: el título provisional de su producto, la situación problemática del tema, la hipótesis sobre sus causas o repercusiones en la esfera social, su interés o motivación por trabajarlo, y el público receptor o beneficiario de la información. Asimismo, debían indicar las razones que justificaban la realización de la investigación periodística, a partir de los criterios del Valor Agregado Periodístico (Pellegrini, Puente, Porath, Mujica, \& Grassau, 2011) como su relevancia, estatus de los implicados, número de implicados o afectados, cercanía geográfica, rareza del caso o por su grado de factor humano.

En este punto, los estudiantes entregaban una primera propuesta de fuentes relevantes o idóneas - por su figuración, credibilidad, responsabilidad o implicación directa en los hechos - en capacidad de dar cuenta del problema y que evidenciaran diversidad de puntos de vista, y realizaban un breve sondeo - estado del arte - sobre lo que hasta el momento se había publicado en medios de comunicación al respecto.

La propuesta grupal se socializó en un consejo de redacción compuesto por los compañeros de la clase. El documento era inmediatamente ajustado, con base en la retroalimentación docente y los aportes de los compañeros y, a continuación, se iniciaban las actividades de reporteo y registro de material para la elaboración de la pieza principal (reportaje audiovisual, sonoro o textual). 


\begin{tabular}{|c|c|c|c|c|c|}
\hline ن & 언 & 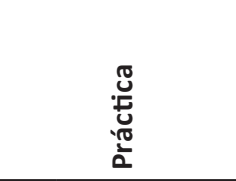 & $\frac{5}{: \frac{5}{U}}$ & 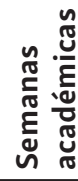 & $\begin{array}{l}\stackrel{0}{\mathrm{~d}} \\
\stackrel{\Delta}{0} \\
\text { ò }\end{array}$ \\
\hline \multirow{5}{*}{$\begin{array}{l}\text { Conceptualización } \\
\text { Teorías de la } \\
\text { tecnología digital } \\
\text { y el fenómeno } \\
\text { convergente }\end{array}$} & \multirow{5}{*}{$\begin{array}{l}\text { Convergencia } \\
\text { Transmedia } \\
\text { Periodismo } \\
\text { transmedia }\end{array}$} & \multirow{5}{*}{$\begin{array}{c}\text { Proposición } \\
\text { tema periodístico } \\
\text { Concepción } \\
\text { de proyecto } \\
\text { transmedia }\end{array}$} & \multirow{5}{*}{$\begin{array}{l}\text { Apropiación conceptual } \\
\text { Aprobación de proyecto } \\
\text { Primer avance de } \\
\text { reporteo }\end{array}$} & 1 & \multirow{3}{*}{ Concepción } \\
\hline & & & & 2 & \\
\hline & & & & 3 & \\
\hline & & & & 4 & \multirow{5}{*}{$\begin{array}{l}\text { Reporteo y } \\
\text { registro de } \\
\text { material }\end{array}$} \\
\hline & & & & 5 & \\
\hline \multirow{5}{*}{$\begin{array}{l}\text { Producción } \\
\text { Apropiación } \\
\text { conceptual e } \\
\text { instrumental para } \\
\text { la creación de } \\
\text { narrativas }\end{array}$} & \multirow{2}{*}{\multicolumn{2}{|c|}{$\begin{array}{c}\text { Web } 2.0 \\
\text { Herramientas y medios sociales } \\
\end{array}$}} & Creación web & 6 & \\
\hline & & & Creación de contenidos & 7 & \\
\hline & \multirow{3}{*}{\multicolumn{2}{|c|}{$\begin{array}{l}\text { Laboratorio de producción de contenidos del } \\
\text { proyecto }\end{array}$}} & multiplataforma & 8 & \\
\hline & & & Entrega de contenidos & 9 & Fdición \\
\hline & & & & 10 & Eurcion \\
\hline Gestión & \multirow{2}{*}{\multicolumn{2}{|c|}{ Plan de acción en redes y espacios físicos }} & \multirow{6}{*}{$\begin{array}{c}\text { Gestión en redes } \\
\text { Actividades en espacios } \\
\text { físicos }\end{array}$} & 11 & Correcciones \\
\hline $\begin{array}{l}\text { Apropiacion } \\
\text { conceptual e }\end{array}$ & & & & 12 & \multirow{4}{*}{ Gestión } \\
\hline instrumental & \multirow{4}{*}{$\begin{array}{l}\text { Creación de } \\
\text { piezas para } \\
\text { plataformas }\end{array}$} & \multirow{4}{*}{$\begin{array}{l}\text { Audiencias } \\
\text { Participación }\end{array}$} & & 13 & \\
\hline para la gestión & & & & 14 & \\
\hline $\begin{array}{l}\text { de contenidos en } \\
\text { redes y realización }\end{array}$ & & & & 15 & \\
\hline $\begin{array}{l}\text { de actividades en } \\
\text { espacios físicos. }\end{array}$ & & & & 16 & Socialización \\
\hline
\end{tabular}

Figura 1. Síntesis programática de la asignatura

Fuente: Elaboración propia.

\section{Etapa 2. Producción de medios y contenidos periodísticos}

En un segundo momento, los estudiantes debían reconocer los medios y herramientas de la Web 2.0, conocer sus usos, funcionalidades y aportes a la narrativa digital con el fin de encauzar la elaboración de sus contenidos. Los estudiantes del grupo trabajaron los contenidos en clase con supervisión docente y lo complementaron en casa como parte de su trabajo autónomo. Al finalizar la etapa, el grupo de trabajo hizo entrega de un sitio web que contiene el reportaje.

\section{Etapa 3. Gestión de medios sociales}

De manera preliminar, los estudiantes trabajaron el concepto de redes sociales y comunidades virtuales que operan a través de los distintos medios sociales. En la práctica, la gestión de redes y comunidades apunta a precisar el público objetivo señalado en la etapa inicial del proyecto y elaborar un plan de acción para intervenir en las redes para atraer a dichos públicos y lograr distintas formas de interacción con el contenido y entre todos miembros de la comunidad. Esto implicó la creación de piezas adicionales para promover la propuesta.

\section{Sistematización de experiencia}

La sistematización de la experiencia fijó como punto de observación dos ejes: observación de proyectos en cada una de las etapas y valoración de la percepción de aprendizaje de once grupos de proyecto que desarrollaron sus propuestas hasta su finalización y que contestaron la encuesta de percepción de aprendizaje. 
1. Caminantes sin camino

2. Bogotá a ciegas

3. Lo que callan los hombres

4. Grooming

5. ¡Vivan los toros!

6. Encendiendo voces

7. El rostro de la calle

8. Vinilos al aire

9. Bogotá accesible

10. Maternidad subrogada

11. El color de las cicatrices
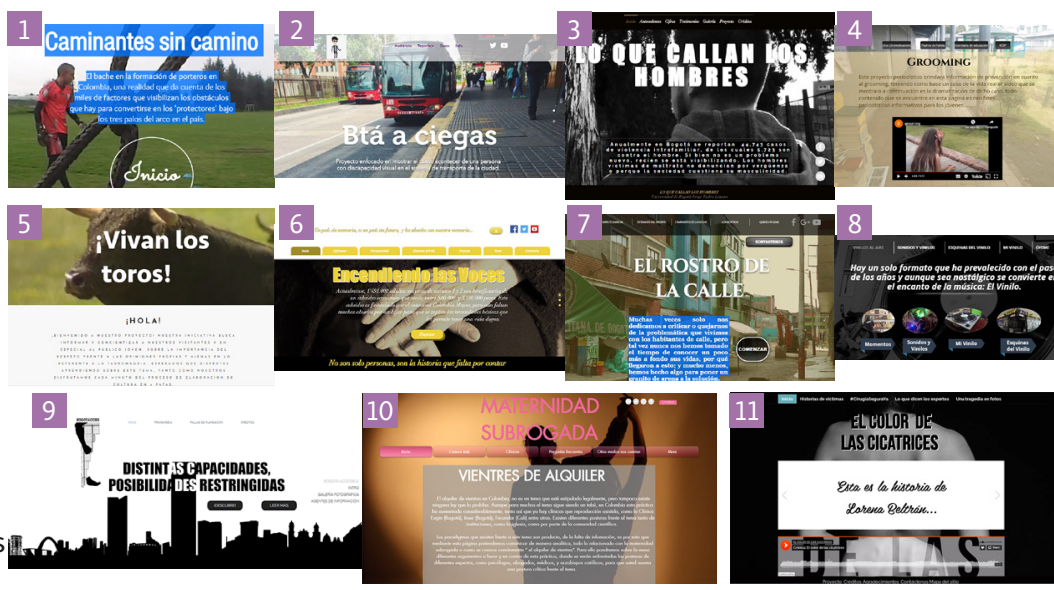

Figura 2. Proyectos elaborados por los estudiantes (2016-2017)

Fuente: Elaboración propia.

\section{Observación de proyectos desarrollados por los estudiantes}

La reconstrucción de la experiencia se inició con la técnica de revisión documental en pantalla del producto final entregado por los estudiantes, presentándola como una fuente de información valiosa para la comprensión del fenómeno estudiado (Hernández-Sampieri, 2014). Para consolidar el registro de antecedentes del material final entregado se elaboró una ficha de recolección de datos. Para su análisis se definieron las categorías por etapas. La ficha se estructuró de la siguiente manera:

- Información básica: título del trabajo y datos de los integrantes.

- Conceptualización: identificación de conceptos teóricos reflejados en la configuración de la propuesta transmedia, la propuesta del tema periodístico y la configuración estructural de la propuesta mediática.

- Producción mediática: observa la creación web como medio principal e integrador del proyecto, así como las piezas que se desarrollaron para articular la historia.

- Gestión de contenidos y audiencias: observa las propuestas y resultados de las estrategias utilizadas por los estudiantes en medios digitales y físicos para involucrar a las audiencias.

\section{Valoración de la percepción de aprendizaje de los estudiantes}

Los datos de este punto se obtuvieron mediante la encuesta aplicada por un formulario de Google a los miembros de los grupos de trabajo. Se compartió el enlace desde el 27 de septiembre de 2018 hasta el 13 de enero de 2019 y fue respondido por 17 estudiantes, de un total de 45 , miembros de los 11 grupos de trabajo presentados en este artículo. En esta encuesta se desarrollaron cinco grupos de preguntas de valoración que otorgan una puntuación de cero (mínimo) a cinco (máximo), más una pregunta abierta (tabla 2). 


\section{Grupos de preguntas}

I. Componente teórico: ¿Considera usted que el componente teórico de la asignatura de Comunicación

y Nuevas Tecnologías II le permitió?:

II. Creación de contenidos: ¿Considera usted que el componente práctico orientado

a la creación de contenidos de asignatura de CNT II le permitió?:

\section{Pregunta a valorar de cero (0) a cinco (5)}

1. Comprender los conceptos de tecnología y convergencia.

2. Comprender el concepto y características de la narrativa transmedia.

3. Reconocer los conceptos mediante el estudio de casos.

4. Diseñar un proyecto periodístico transmedia a partir de la teoría y los casos estudiados.

5. Explorar los diferentes tipos de medios sociales de la Web 2.0, sus características y usos.

6. Comprender el potencial narrativo de los diferentes medios y herramientas de la Web 2.0.

7. Practicar la producción de sitios web y la creación de contenidos multimedia a través de diferentes herramientas de la Web 2.0.

8. Hacer un uso pertinente de los medios para contar historias tomando como base los conceptos teóricos.

9. Adquirir la competencia de emplear cualquier medio de la Web 2.0 para generar medios y contenidos periodísticos.

III. Gestión de contenidos en medios sociales: ¿Considera usted que el componente práctico orientado a la gestión de contenidos en redes sociales a través de medios sociales de la asignatura CNT II le permitió?:

IV. Aportes de la asignatura a su formación de periodista: ¿Considera usted que la asignatura de Comunicación y Nuevas Tecnologías II le permitió?:
10. Identificar y practicar formas de atracción de audiencias a través de medios sociales.

11. Identificar y practicar formas de involucramiento de audiencias a través de medios sociales.

12. Identificar y practicar formas de involucramiento (participación-acción) en medios físicos.

13. Aproximarse a una práctica del periodismo y formas de producción a partir de la narrativa transmedia.

14. Reconocer y aplicar diferentes estrategias y técnicas narrativas de la convergencia para aplicar al periodismo.

15. Aportar a la comprensión y solución de problemas sociales.

16. Fortalecer sus competencias periodísticas.

17. Fortalecer sus competencias instrumentales basadas en la Web 2.0.

18. Fortalecer su capacidad de trabajo en equipo.

V. Aportes

19. ¿Qué recomendaciones haría usted a esta asignatura para fortalecer el proceso de enseñanza?

Tabla 2. Preguntas encuesta percepción de aprendizaje de los estudiantes

Fuente: Elaboración propia.

\section{RESULTADOS}

\section{Observación de proyectos}

Los proyectos periodísticos transmedia desarrollados por los estudiantes evidenciaron un interés por abordar problemáticas de la realidad y de actualidad para comprender su naturaleza e impacto de cara a la generación de contenidos que ayuden a dar mayores elementos de reflexión. En concordancia con los abordajes teóricos, plantearon estructuras investigativas, mediáticas y multiplataforma pertinentes, viables para gestionar el contenido y sus narrativas, así como para gestionar los medios sociales por los cuales buscarían poner en circulación sus mensajes y actividades a realizar con sus nacientes comunidades. 
La forma en la que se articularía la historia a través de tales medios y plataformas fue el aspecto que generó más incertidu mbre dada la amplia diversidad de medios, lenguajes y tácticas narrativas, y sus posibilidades de conexión para articular el planteamiento periodístico. El realizar una inscripción planteando puntos de proyecto en el marco de un consejo de redacción con los compañeros y el profesor ayudó a concretar el tema de investigación desde una etapa muy temprana del curso, establecer rutinas de organización y producción entre los grupos. Luego de esta primera presentación en el consejo de redacción, todas las propuestas fueron sometidas a correcciones.

En la etapa de producción mediática, los estudiantes se aproximaron teórica y prácticamente a los medios y herramientas de la Web 2.0 para la generación de contenidos y su gestión en las redes sociales. En los talleres, los jóvenes reconocieron los instrumentos y trabajaron en la creación de cuentas nuevas de su proyecto, en donde identificaron su potencial narrativo y la posibilidad de editar, publicar y compartir. A nivel de sitio web, en las versiones iniciales, los problemas más recurrentes entre los estudiantes tuvieron que ver con la configuración de la estructura general del sitio y con la conexión narrativa de los contenidos complementarios a la pieza principal. Se detectó que, en general, faltó desarrollar más la propuesta de contenido, lo que sugiere que es necesario profundizar en una disciplina de producción de experiencias de usuario (Garrett, 2011) en la etapa que define y elabora los contenidos a publicar.

En la producción de los sitios, se observó que la calidad del material multimedia recolectado, proveniente de los equipos móviles y sensiblemente dependiente de las condiciones de registro, de las técnicas empleadas y de los procesos de edición, afectó seriamente la legibilidad y la credibilidad de los contenidos. Por tal motivo, resulta imperativo en experiencias futuras implementar sesiones de práctica en campo con fuentes, locaciones y recursos técnicos, para mitigar los inconvenientes técnicos y así mejorar la calidad. Otro error común detectado fue la tendencia de los estudiantes a elegir herramientas y formas narrativas que no son pertinentes al tipo de material recolectado, y viceversa, privilegiando en la mayoría de los casos más la capacidad efectista del medio interactivo que el potencial narrativo. Por ello, se hace necesario que los estudiantes tengan acceso a casos de referencia que les permitan tener mayor criterio y certeza.

Como el sitio web debía ser el medio principal de la experiencia periodística, actuando como aglutinador de las diferentes temáticas, narrativas y experiencias con audiencia, no es de extrañar que Wix y Webbly hayan sido algunas de las herramientas 2.0 de creación de sitios más utilizadas en los proyectos. Por su parte, YouTube fue utilizado en ocho de los productos para articular el reportaje como 
narrativa principal. Sin embargo, también se utilizó para agregar testimonios, los registros de las actividades realizadas en espacios físicos con audiencias, y para elaborar contenido específico para compartir en redes sociales. Algunos casos lo emplearon para crear clips que planteaban preguntas que se resolvían a partir de testimonios.

La herramienta TimelineJS —desarrollada por la Fundación Knight— para construir cronologías hipermediales fue utilizada sin mayor inconveniente desde el punto de vista técnico, pero sí evidenció la necesidad de trabajar más con el estudiante el potencial y uso pertinente de esta narrativa. Lo anterior, debido a que en algunos casos el componente temporal fue descuidado y terminó convirtiendo la propuesta en una secuencia de contenidos desarticulados, y porque la interfaz gráfica de usuario pudo ser mejor aprovechada. En igual caso, el uso de JuxtaposeJS, para la superposición interactiva de imágenes, evidenció la necesidad de explotar más la capacidad comparativa de imágenes sobre el mismo plano para tener oportunidad de contrastar y apreciar los cambios, diferencias y similitudes entre el antes y después en un contexto gráfico. La simplicidad de esta funcionalidad exige un mayor tratamiento y precisión de las imágenes.

StoryMapJS, como relato hipermedia que plantea la georreferenciación secuencial, se aplicó en algunos casos de manera correcta bajo la modalidad de recorrido o rutas, y en otros casos, subutilizada para realizar simples referencias que pudieron ser tratadas con un mapa convencional. La georreferenciación de Google Maps fue recurrente en los proyectos y se concentró en la personalización de mapas con marcadores que señalaban establecimientos o zonas de afección en relación con sus temáticas. Su despliegue, sin embargo, habría podido ser más destacado en el espacio de la interfaz para mejorar el campo de consulta, y también explotar su potencial implementando marcadores personalizados con contenido multimedia que aportaran mayores elementos informativos, o con un mejor trabajo de titulación y descripción.

Playbuzz fue empleado para proveer un juego de trivia interactivo en donde el usuario es desafiado responder en línea las preguntas sobre los contenidos expuestos en la narrativa principal. Se identificó también la implementación de Soundcloud, que tuvo tres usos específicos: en el primer caso apuntó a realizar la crónica sonora; en el segundo, a presentar testimonios complementarios en diferentes puntos de la narrativa, y, en el tercero, como podcasts de corta duración, que también fueron emitidos en la emisora virtual de la universidad. Algo similar ocurrió con Spreaker, que se empleó exclusivamente para la presentación de una serie podcast.

Piktochart e Infogram fueron plataformas usadas para exponer infografías que sintetizan cifras obtenidas de estudios realizados por fuentes oficiales o académicas, 
como recurso para mejorar la comprensión. Sin embargo, las características de los modelos predefinidos en las plataformas, si bien representan una ayuda en el diseño, llevan a que se pierda la esencia de la información, debido a que las plantillas no llegan a ser personalizadas estéticamente para responder a la temática del proyecto. Por ello, es fundamental reflexionar sobre el uso, pertinencia y aporte de estos modelos en relación con el contenido.

Los medios sociales Facebook, Twitter e Instagram fueron agregados como enlace y en casos puntuales para incorporar contenido creado por audiencias. En lo correspondiente a la tercera etapa, relativa a la gestión de contenidos en medios sociales, los estudiantes desarrollaron actividades de atracción e involucramiento a través de medios sociales y en espacios físicos, en la mayoría de los casos de manera articulada (exclusivamente en el medio social, exclusivamente en el escenario físico, o de manera combinada entre análogo y digital).

La gestión de los medios sociales se realizó con el objetivo de propiciar la conformación y dinamización de comunidades virtuales, teniendo como norte la búsqueda de la participación de la audiencia mediante las distintas formas de interacción que proveen tales medios. Las estrategias derivaron en un ejercicio planificado, cuyas principales acciones fueron la entrega periódica de nuevo material multimedia para mantener al usuario actualizado, presentándole informaciones relacionadas o los avances del proyecto. Por otro lado, se invitó a líderes y organizaciones digitales clave en las temáticas desarrolladas para convertirlos en seguidores, multiplicadores, aliados o participantes activos de la comunidad.

También se formularon preguntas o encuestas a la comunidad y se la invitó a asistir a las actividades programadas, así como a fomentar la recomendación para aumentar el alcance. Dependiendo de la temática y de los públicos objetivos, los grupos procuraron instar a las audiencias a la acción, organizando actividades tradicionales o disruptivas con el fin de acercar a sus públicos a la problemática. Algunas de estas fueron la realización de recitales al aire libre, debates públicos en auditorio, juegos, experimentos sociales, creación artística como murales o dibujos en papel, o recorridos de reconocimiento. En algunos casos, estas actividades fueron convocadas desde las redes sociales, que también fueron un instrumento para recuperar las participaciones, entre otras prácticas.

\section{Resultados de la encuesta de valoración de los estudiantes frente al desarrollo de la asignatura}

Con un puntaje de 4,4 , sobre 5 , equivalente a $88 \%$ de aceptación sobre el componente teórico del curso, los estudiantes consideraron que este les permitió comprender el concepto y las características de la narrativa transmedia. Con la misma puntuación manifestaron haber comprendido los conceptos de tecnología y convergencia y que, a partir de esto, haya sido posible diseñar un proyecto 
periodístico transmedia. Con una valoración de 4,1 (82\%), calificaron haber reconocido los conceptos a través del estudio de casos. Respecto del componente práctico, con una puntuación de 4,1 los estudiantes afirman que fue posible explorar los diferentes tipos de medios sociales de la Web 2.0. Con calificación de 4,2 manifiestan que comprendieron el potencial narrativo de los diferentes medios y herramientas de las Web 2.0. Con una puntuación de 4,4, manifestaron haber tenido la posibilidad de practicar la producción de sitios web y la creación de contenidos multimedia a través de diferentes herramientas de la Web 2.0. En el mismo grado, consideran haber hecho un uso pertinente de los medios para contar historias, tomando como base los conceptos teóricos y, de igual modo, haber logrado adquirir la competencia de emplear cualquier medio de la Web 2.0 para generar medios y contenidos periodísticos.

En lo correspondiente al trabajo de gestión de contenidos de redes sociales, los estudiantes puntuaron con 4,0 el haber podido identificar y practicar formas de atraer audiencias a sus redes. Con 4,1 valoraron el haber podido identificar y practicar formas de involucrarlas a sus actividades propuestas, y con 3,9, el haberlas involucrado (convocado) a espacios físicos. Sobre la asignatura, los estudiantes puntúan con 4,3 el aporte que tuvo esta materia al permitirles aproximarse a la práctica periodismo y a las formas de producción a partir de la narrativa transmedia. Con 4,6, afirman que les permitió reconocer y aplicar diferentes estrategias y técnicas narrativas de la convergencia para aplicar al periodismo. Con valoraciones de 4,1 y 4,2 respectivamente, la asignatura también les permitió hacer sus aportes a la comprensión y solución de problemas sociales y fortalecer sus competencias periodísticas. Asimismo, con una puntuación de 4,3 expresaron que este abordaje les permitió fortalecer sus competencias instrumentales basadas en la Web 2.0 y su capacidad de trabajo en equipo.

En lo que respecta a la quinta parte del instrumento, en donde se invitó los estudiantes a hacer sus aportes para mejorar el proceso de enseñanza, los jóvenes manifestaron aspectos positivos y otros que deben tenerse en cuenta para mejorar la propuesta ABP. En primer lugar, ven como completa y pertinente la propuesta pedagógica, en donde el acompañamiento fue importante para cumplir con el objetivo. Consideran que la metodología que integra la teórica y práctica fue suficiente y sirvió para dar impulso al proyecto, dándoles la capacidad de reconocer y aplicar distintas estrategias comunicativas. Asimismo, aseguran que la asignatura pone a prueba su creatividad a la hora de realizar y volver transmedia el proyecto, y consideran que es un buen ejercicio que aporta mucha experiencia para la vida profesional.Sin embargo, algunos de los estudiantes llaman la atención sobre el componente teórico, que consideran excesivo y monótono cuando se trata en la primera etapa, lo que ven como contraproducente respecto de los tiempos de aplicación del concepto al 
producto y a su gestión, por lo que sugieren que esta etapa teórica sea reajustada y todavía más conectada a sus proyectos. Además, plantean la necesidad de contar con mayores referentes casuísticos que les permitan delinear y enriquecer sus propuestas.

En cuanto a lo práctico, los estudiantes consideran importante que la apropiación instrumental se realice también desde el comienzo del curso y no solo con las propuestas desde la Web 2.0, sino con las aplicaciones de escritorio que emplean las empresas. Consideran que es importante que la producción de contenidos se aumente en horas de clase y logre involucrarlos más con su entorno.De igual manera, desean profundizar más en el campo estratégico, así como en la promoción de sus productos para darlos a conocer. Buscan particularmente enfocarse en el trabajo bajo presión, para que sea coincidente con lo que se experimenta en un ambiente laboral real, debido a que identifican que la forma de producción en los medios es acelerada y requiere de la mayor y mejor capacidad de reacción por parte del profesional.

\section{DISCUSIÓN}

En líneas generales, los resultados de la experiencia señalan que el ABP es un instrumento pedagógico clave para la enseñanza del Periodismo Transmedia, que permitió que la estructura de la asignatura cumpliera con los objetivos de propiciar un mejor proceso y experiencia de aprendizaje significativo para los estudiantes. Esto sugiere que esta propuesta se siga desarrollando en el futuro y se realicen los respectivos ajustes metodológicos que permitan optimizar el proceso y aumentar el conocimiento, la motivación y el nivel de experticia para la realización de sus trabajos.

La configuración de los proyectos muestra que plantear un sistema multiplataforma para contar una historia no es en principio una tarea difícil, pero que sí implica un conocimiento amplio de los medios y narrativas y, sobre todo, un gran esfuerzo creativo y razonado para hilar las historias por las vertientes del circuito mediático, que procure mantener la atención y participación de la audiencia.

En materia de producción técnica, el aprendizaje de los instrumentos es rápido, pero requiere de un mayor trabajo en lo que concierne al concepto del contenido, ya que se evidencia que en algunos casos la apropiación instrumental es buena y clara, pero su contenido es deficiente y está desviado de su propósito o función narrativa. Esto sugiere que, desde etapas más tempranas de la formación de periodistas transmedia, ya exista un antecedente que afiance su criterio estético y comunicacional. Aunque la valoración de los estudiantes fue positiva, también queda claro que existen muchos frentes por cubrir para llegar al tipo de conocimiento, habilidad y producto 
que los participantes esperan aprender a realizar. Es por ello que este ejercicio debe permanentemente registrar las iniciativas, buenas prácticas o situaciones complejas que conduzcan a implementar mejoras a la propuesta pedagógica.

A la fecha de publicación de este artículo, la asignatura no se adelanta debido a la actualización del plan de estudios. No obstante, la configuración y antecedente de la clase de Comunicación y Nuevas Tecnologías II edificó el contenido programático vigente de la primera de dos asignaturas de Convergencia en el Programa de Comunicación Social - Periodismo de la Universidad de Bogotá Jorge Tadeo Lozano.

\section{CONCLUSIONES}

Como puede observarse de la iniciativa pedagógica planteada y la experiencia aquí expuesta, se puede concluir que la formación de periodistas transmedia en el marco del modelo de Aprendizaje Basado en Problemas permite obtener un aprendizaje significativo. Esto es así en la medida en que el estudiante tenga, en cada etapa de proyecto, el reto de exigirse en el pensar y el hacer para construir un trabajo con los elementos necesarios que le permitan contar historias y atraer a las audiencias, enfrentando y comprendiendo de manera temprana los desafíos que Rost y Bergero (2012) advierten para el periodismo en el ámbito convergente.

También queda claro que el periodismo transmedia no se resuelve únicamente con abordajes bibliográficos o magistrales, sino que resulta necesario que el estudiante ejercite la concepción, estructuración y ejecución de proyectos narrativos para poder consolidar el conocimiento útil para su vida laboral; un trabajo que tendrá mayor contundencia en la medida en que las temáticas a tratar sean del gusto de los estudiantes y tengan un propósito social claro.

En medio de la complejidad del contexto convergente, la tarea del docente debe ser tan comprometida como la de sus estudiantes, ya que, en su labor de guía, asesor y crítico, debe encauzar las ideas y llevar a hechos concretos la teoría, así como llamar la atención a los grupos de trabajo sobre las posibilidades, oportunidades, estrategias, instrumentos y buenas prácticas productivas para dar consistencia a las propuestas.

Para el desarrollo de proyectos periodísticos transmedia, además de reconocer el ecosistema mediático que nos explica Carlos Scolari (2013), es fundamental entrar en contacto directo con los medios que lo componen y comprender en detalle, a través de la práctica, sus dinámicas y particularidades para así establecer relaciones - sinergias - entre cada una de ellas, reduciendo el nivel de construcciones fragmentadas y desconectadas entre sí. Esto supone también ahondar en el detalle de las posibilidades narrativas que permite cada medio. 
Resulta de gran valor que los estudiantes hayan tenido, previa o paralelamente, la oportunidad de desarrollar la producción de narrativas periodísticas, audiovisuales, digitales y sonoras, ya que el conocimiento en estas áreas facilita el proceso de producción y edición de los proyectos. De acuerdo con lo anterior, es importante instar a que estas producciones se conecten y apoyen activamente con un medio, laboratorio o unidad productiva extra clase, con el objetivo de que los estudiantes tengan un escenario adicional para dar continuidad al desarrollo de los proyectos y el trabajo autónomo se aproveche en un ambiente idóneo. Este sería un escenario que, además, podría entregar al estudiante más instrumentos para el cumplimiento de sus objetivos.

Es importante señalar que la producción periodística transmedia sigue siendo un terreno en construcción, altamente cambiante, que no cuenta con fórmulas exactas ni ingredientes específicos. Cada propuesta es una experiencia nueva y única que se define según la temática, las características de sus audiencias, los presupuestos, etc., y, particularmente, de lo que se quiere obtener de ellas. En cualquiera de sus configuraciones, las propuestas representan para sus participantes un ejercicio que demanda de bastante conocimiento, creatividad, coordinación y disciplina de trabajo.

\section{REFERENCIAS}

Aparici, R., García-Marín, D., \& Díaz-Delgado, N. (2019). Vampiros en la Red. El robo de la cultura juvenil (Vampires on the Web. The exploitation of youth culture). Revista Latina de Comunicación Social, (74), 197-213. https://doi.org/10.4185/RLCS-2019-1327

Banco Mundial. (2017). Personas que usan Internet (\% de la población) (Internet Users \% Population) (data set). Retrieved from https://datos.bancomundial.org/indicador/ IT.NET.USER.ZS? contextual=default\&end=2017\&locations $=$ CO\&start $=1990 \&$ view $=$ chart

Bermúdez, C. (2018). Lógica práctica y lógica teórica en la sistematización de experiencias educativas (Practical Logic and Theoretical Logic in the Systematization of Educational Experiences). Pedagogía y Saberes, (48), 141-151. https://doi.org/10.17227/pys.num48-7379

Camargo, A., Vieira Miranda, G., \& Magnon, A. (2019). Convergencia mediática y cultura participativa: la posibilidad de nuevas relaciones entre los agentes sociales en el campo de la comunicación y las nuevas tecnologías (Media Convergence and Participatory Culture: The Possibility of New Relationships between Social Agents in the Field of Communication and New Technologies). Anuario Electrónico de Estudios en Comunicación Social "Disertaciones", 12(1), 75-87. https://doi.org/10.12804/revistas.urosario.edu.co/disertaciones/a.6071 
Carranza, M. \& Caldera, J. (2018). Percepción de los Estudiantes sobre el Aprendizaje Significativo y Estrategias de Enseñanza en el Blended Learning (Perception of Students on Meaningful Learning and Teaching Strategies in Blended Learning). REICE. Revista Iberoamericana sobre Calidad, Eficacia y Cambio en Educación, 16(1), 73-88. https://doi.org/10.15366/reice2018.16.1.005

Casa, M., Huatta, S., \& Mancha, E. (2019). Aprendizaje Basado en Problemas como estrategia para el desarrollo de competencias en estudiantes de educación secundaria (Problem Based Learning as strategy for the development of competences in secondary education students).Comuni@cción, 10(2),111-121. https://doi.org/10.33595/2226-1478.10.2.383

Costa, C. \& Piñeiro, T. (2012). Nuevas narrativas audiovisuales: multiplataforma, crossmedia y transmedia: El caso de Águila Roja (RTVE) (Audiovisual narratives: multi-platform, crossmedia and transmedia: The Aguila Roja Case). ICONO14, 10(2), 102-125. https://doi.org/10.7195/ri14.v10i2.156

Datareportal. (2020). Global Digital Insigths: Digital 2020: Colombia. Retrieved from https:// datareportal.com/reports/digital-2020-colombia

Departamento Administrativo Nacional (DANE). (2017). Estimaciones 1985-2005 y Proyecciones 2005-2020 nacional y departamental desagregadas por sexo, área y grupos quinquenales de edad (Estimates 1985-2005 and projections 2005-2020 national and departmental disaggregated by sex, area and five-year age groups) (Excel file). Retrieved from http://www.dane.gov.co/files/investigaciones/poblacion/seriesp85_20/ EstimacionesProyecciones1985_020.xls

Garrett, J. J. (2011). The Elements of User Experience: User-Centered Design for the Web and Beyond (Second Edition ed.). New Riders.

Hernández-Sampieri, R. (2014). Metodología de la Investigación (6a ed.) (Research methodology, 6th ed.). Mexico City, Mexico: McGraw Hill Education.

Jenkins, H. (2008). Convergence Culture: La cultura de la convergencia de los medios de comunicación (Convergence Culture: Where Old and New Media Collide). Buenos Aires, Argentina: Ediciones Paidós Ibérica, S.A.

Larrondo Ureta, A., Canavilhas, J., Fernandes Teixeira, J., Martins, G. L., Meso Ayerdi, K., Peña Fernández, S., Pérez Dasilva, J., \& Zamith, F. (2020). Innovación educativa para la internacionalización y la convergencia de la enseñanza del ciberperiodismo en Iberoamérica (Educational innovation for the internationalization and convergence of university teaching of online journalism in Ibero-America). Anàlisi, (62), 35-56. https:// doi.org/10.5565/rev/analisi.3264

López-Carril, S., Villamón-Herrera, M., \& Añó-Sanz, V. (2019). Conceptualización de los medios sociales (Conceptualization of social media). RETOS- Nuevas Tendencias en Educacion Fisica, Deporte y Recreacion, (36), 578-583. https://doi.org/10.47197/retos.v36i36.68572

Luy-Montejo, C. (2019). El Aprendizaje Basado en Problemas (ABP) en el desarrollo de la inteligencia emocional de estudiantes universitarios (Problem Based Learning (PBL) in the Development of Emotional Intelligence of University Students). Propósitos $y$ Representaciones, 7(2), 353-383. https://doi.org/10.20511/pyr2019.v7n2.288 
Maldonado-Pérez, M. (2008). Aprendizaje Basado en Proyectos Colaborativos. Una experiencia en educación superior (Learning based on collaborative projects. An experience in higher education). Laurus. Revista de Educación, 14(28), 158-180.

Méndez Urresta, E. M., Méndez Urresta, J. B., \& Méndez Carvajal, V. C. (2017). El aprendizaje basado en problemas como vía para el desarrollo de competencias en Educación Superior (The learning based in problems like a way for the development of competences in higher education). Revista Conrado, 13(60), 21-25. Retrieved from https://conrado.ucf.edu.cu/ index.php/conrado/issue/view/38

Ministerio de la Tecnologías de la Información y las Comunicaciones (Mintic). (2017). Primera Gran Encuesta TIC/2017. Estudio de acceso, uso y retos de las TIC en Colombia (First Great ICTs Survey/2017. Study of access, use and challenges of ICTs in Colombia). Bogota, Colombia: Torre Blanca. Retrieved from https://colombiatic.mintic.gov.co/679/articles-74002_ cartilla_resumen.pdf

Ministerio de la Tecnologías de la Información y las Comunicaciones (Mintic). (2020). Telefonía Móvil (Mobile Telephony) (data set). Retrieved from https://colombiatic.mintic. gov.co/679/w3-propertyvalue-47274.html

Moreno, I. (2012). Narrativa Hipermedia y Transmedia (Hypermedia and transmedia narrative). In V. Perales Blanco (Ed.), Creatividad y discursos hipermedia (Creativity and hypermedia discourses) (pp. 21-40). Murcia, Spain: Edit.um. https://doi.org/10.13140/RG.2.1.2538.1602

Nieto, N. (2017). Transmediaciones en la producción de contenidos periodísticos especializados en economía (Transmediations in the journalistic content output in specialized economic media). Cuadernos.Info, (40), 219-233. https://doi.org/10.7764/cdi.40.1037

Pellegrini, S., Puente, S., Porath, W., Mujica, C., \& Grassau, D. (2011). Valor agregado periodístico: La apuesta por la calidad de las noticias (Journalistic Added Value: The bet for the quality of news). Santiago, Chile: Ediciones Universidad Católica de Chile.

Pérez-Rodríguez, A. (2020). Homo sapiens, homo videns, homo fabulators. La competencia mediática en los relatos del universo transmedia (Homo sapiens, homo videns, homo fabulators. Media competence in the narratives of the transmedia universe). ICONO14, 18(2), 16-34. https://doi.org/10.7195/ri14.v18i2.1523

Pratten, R. (2015). Getting Started in Transmedia Storytelling: A Practical Guide for Beginners (2nd ed.). CreateSpace Independent Publishing Platform.

Renó, D. \& Flores, J. (2018). Periodismo transmedia (Transmedia journalism). Madrid, Spain: Editorial Ria.

Rost, A. \& Bergero, F. (2012). Periodismo en contexto de convergencias (Journalism in the context of convergences). Rio Negro, Argentina: Publifadecs.

Sarsa, J. (2014). El perfil prosumidor de los estudiantes en la web 2.0 (The Profile of the students as prosumers in web 2.0). Journal for Educators, Teachers and Trainers, 5(2), 74-87. Retrieved from https://jett.labosfor.com/article_803.html 
Scolari, C., Manuel, J., \& Mar, G. (2012). Narrativas transmediáticas en España: cuatro ficciones en busca de un destino cross-media (Transmedia Storytelling in Spain: four fictions searching for their cross-media destiny). Comunicación y Sociedad, 25(1), 137-163. Retrieved from https://revistas.unav.edu/index.php/communication-and-society/article/view/36179

Scolari, C. (2013). Narrativas Transmedia: Cuando todos los medios cuentan (Transmedia Narratives: When all media counts). Barcelona, Spain: Deusto - Centro Libros PAPF.

Scolari, C. A. (2016). Alfabetismo transmedia: estrategias de aprendizaje informal y competencias mediáticas en la nueva ecología de la comunicación (Transmedia literacy: informal learning strategies and media skills in the new ecology of communication). Telos, (103), 13-23. Retrieved from https://telos.fundaciontelefonica.com/archivo/numero103/

Scolari, C. A. \& Establés, M. J. (2017). El ministerio transmedia: expansiones narrativas y culturas participativas (The Transmedia Ministry: Narrative Expansions and Participatory Cultures). Palabra Clave, 20(4), 1008-1041. https://doi.org/10.5294/ pacla.2017.20.4.7

Vázquez-Herrero, J., González-Neira, A., \& Quintas-Froufe, N. (2019). La audiencia activa en la ficción transmedia: plataformas, interactividad y medición (Active Audience in Transmedia Fiction: Platforms, Interactivity and Measurement). Revista Latina de Comunicación Social, (74), 73-93. https://doi.org/10.4185/RLCS-2019-1322

Villanueva-Mansilla, E. (2017). Convergencia mediática: lecciones y preguntas desde la experiencia peruana (Media convergence: lessons and questions from the Peruvian experience). Revista de Comunicación, 16(1), 166-191. Retrieved from https://revistadecomunicacion.com/article/view/1013

\section{SOBRE EL AUTOR}

NELSON NIETO BORDA, comunicador social y periodista de la Universidad Central (Colombia), magíster en Comunicación y Periodismo Digital de la Universidad Mayor (Chile) y estudiante del Doctorado en Comunicación de la Universidad Nacional de la Plata (Argentina), desde el cual ha profundizado en estudios sobre transmedia y nuevas audiencias.

(iD https://orcid.org/0000-0001-5049-9899 